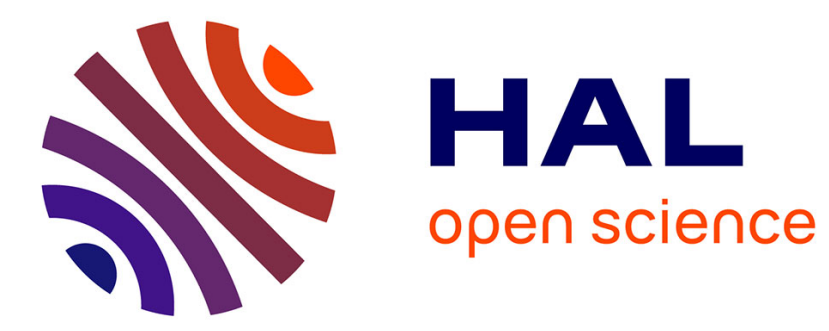

\title{
Experimental 5G New Radio integration with VLC
}

Lina Shi, Wei Li, Xun Zhang, Yue Zhang, Gaojie Chen, Andrei Vladimirescu

\section{To cite this version:}

Lina Shi, Wei Li, Xun Zhang, Yue Zhang, Gaojie Chen, et al.. Experimental 5G New Radio integration with VLC. 2018 25th IEEE International Conference on Electronics, Circuits and Systems (ICECS), Dec 2018, Bordeaux, France. pp.61-64, 10.1109/ICECS.2018.8617851 . hal-02333638

\section{HAL Id: hal-02333638 \\ https://hal.science/hal-02333638}

Submitted on 25 Oct 2019

HAL is a multi-disciplinary open access archive for the deposit and dissemination of scientific research documents, whether they are published or not. The documents may come from teaching and research institutions in France or abroad, or from public or private research centers.
L'archive ouverte pluridisciplinaire $\mathbf{H A L}$, est destinée au dépôt et à la diffusion de documents scientifiques de niveau recherche, publiés ou non, émanant des établissements d'enseignement et de recherche français ou étrangers, des laboratoires publics ou privés. 


\title{
Experimental 5G New Radio integration with VLC
}

\author{
Lina Shi ${ }^{1}$, Wei $\mathrm{Li}^{2}$, Xun Zhang ${ }^{1}$, Yue Zhang ${ }^{3}$, Gaojie Chen $^{3}$, Andrei Vladimirescu ${ }^{1}$ \\ ${ }^{1}$ Institut supérieur d'électronique de Paris, Paris, France \\ ${ }^{2}$ Viavi Solutions, Stevenage, UK \\ ${ }^{3}$ Department of Engineering, University of Leicester, UK \\ *Contact: lina.shi@isep.fr
}

\begin{abstract}
In this paper, integration of 5G New Radio (5G NR) with a Visible Light Communication (VLC) downlink architecture is proposed. This scheme combines two complementary wireless technologies: upcoming 5G NR and VLC to offer indoor enhanced wireless hybrid access able to provide each User Equipment (UE) with very high data rate and positioning support. The data transmission of the 5G NR frame over VLC has been implemented. This represents a novel approach to transmitting $5 \mathrm{G}$ NR over VLC by hardware experimentation based on Universal Software Radio Peripheral (USRP). The experiment results show that the proposed scheme with Quadrature Phase Shift Keying (QPSK) mapping achieves a data rate of $14.4 \mathrm{M}$ bits/s and an Error Vector Magnitude (EVM) of $4.78 \%$ for a $55 \mathrm{~cm}$ free space transmission span.
\end{abstract}

Keywords-5G New Radio; Visible Light Communication; Software Defined Networks; USRP

\section{INTRODUCTION}

In recent years, mobile data traffic has been growing at an accelerating rate. The latest data from Cisco predicts that the mobile data traffic will reach approximately 24 Exabyte per month by 2019 [1]. $80 \%$ of this growth is generated in indoor environments where wireless traffic increases $20 \%$ faster than outdoors each year [2]. Meanwhile, in consumer electronics the top technology trends focus on the number of network interfaces, screen sizes and resolution, which are increasing to meet the demand for higher quality of video experience for different User Equipment (UE). Since video streaming is the media that uses the most network capacity, there is a consequent increase in demand for network resources to deliver these video services and distribute them within homes. With this growing capability and demand, the indoor interference and congestion issues become more and more prominent.

Actually, more than $75 \%$ of the wireless data traffic is using Wi-Fi indoor access points, while classical cellular networks cover the remaining. But these two wireless technologies are facing spectrum congestion, especially Wi-Fi, when a huge number of UE are present within a confined indoor space and requesting high traffic services, such as in conference, congress centers or shopping malls.

Therefore, as the new air interface for the next generation of communication networks, 5G NR is expected to meet the aforementioned requirements to achieve a high-capacity, highdata-rate and low-interference data service in indoor environments [3-5].

Visible Light Communication (VLC) is gaining more and more interest due to its very high throughput performance in indoor and its security advantages for both privacy and health
[6][7]. In fact, a largely so-far neglected part of the spectrum is the optical window spanning from infrared into the visible part of the spectrum on to ultraviolet frequencies. We believe that these frequencies are of great importance for future mobile communication solutions beyond $5 \mathrm{G}$, not only due to the huge amount of unregulated spectrum offered, but also because of an existing base of mature technology components stemming from fiber and free-space optical communications and synergies, which can be exploited by building on existing illumination infrastructures. Moreover, LEDs are becoming the predominant light source and could transform in the coming years any light bulb into a VLC access point, which not only reduces a lot the extra-wired infrastructure needed to implement the backhaul network, but also diminishes drastically the user. Electromagnetic Field (EMF) exposure that today explodes with Wi-Fi becomes a health concern, as proven by the Loi Abeille promulgated in 2015 in France [8]. To summarize, VLC is deemed to be a desirable technology for indoor wireless access technology in future $5 \mathrm{G}$ networks.

Based on aforementioned reasons this paper is the first work to consider $5 \mathrm{G}$ NR and VLC, which can provide each UE with high data rate, seamless $5 \mathrm{G} / \mathrm{Li}-\mathrm{Fi}$ connectivity and fine localization with low latency. The main contributions of this work are the following:

- A 5G NR integration with VLC downlink scheme to be implemented on USRP. As far as we know, this is the first experimental implementation proof of $5 \mathrm{G}$ NR over VLC transmission based on USRP.

- It provides each UE with data rate of $14.4 \mathrm{M}$ bits/s and the EVM of $4.78 \%$ for a $55 \mathrm{~cm}$ free-space transmission span. The positioning support will be considered in our future work.

The rest of this paper is organized as follows: section II details a VLC downlink architecture with 5G NR integration. An experimental testbed and measurement results are described in detail in section III. Section IV concludes this paper.

\section{5G NR INTEGRATION WITH VLC DOWNLINK ARCHITECTURE}

In this section the proposed $5 \mathrm{G}$ NR over VLC downlink scheme is presented first. Second, the adaptation of $5 \mathrm{G} \mathrm{NR}$ modulation and frame structure is described to meet the requirements of VLC transmission.

A diagram of the proposed downlink scheme is illustrated in Fig. 1. In this diagram, the Radio Access Network (RAN) is responsible for data and signal transmission between the 
Ethernet and UEs. RAN uses a $10 \mathrm{G}$ Common Public-Radio Interface (eCPRI) Ethernet ring to access the $5 \mathrm{G}$ core network.

The Ethernet ring interconnects an Upper-Layer 1 processing unit to up to six Remote Radio Light Heads (RRLHs) controllers. Each RRLH hosts a Lower-Layer 1 processor used for precoding, Inverse Fast Fourier Transform (IFFT), Cyclic Prefix (CP) insertion, windowing and symbol overlapping in the downlink. The Upper-Layer 1 is the physical layer central unit. This unit major tasks include channel encoding, decoding and scrambling.

The main function of the RRLH controller is the transmission of information in the visible light spectrum. Each RRLH generates the low intermediate frequency (IF) signals for 8 VLC Multi-Input Single-Output (MISO) modules by using a 1 to 8 RF splitter [9].

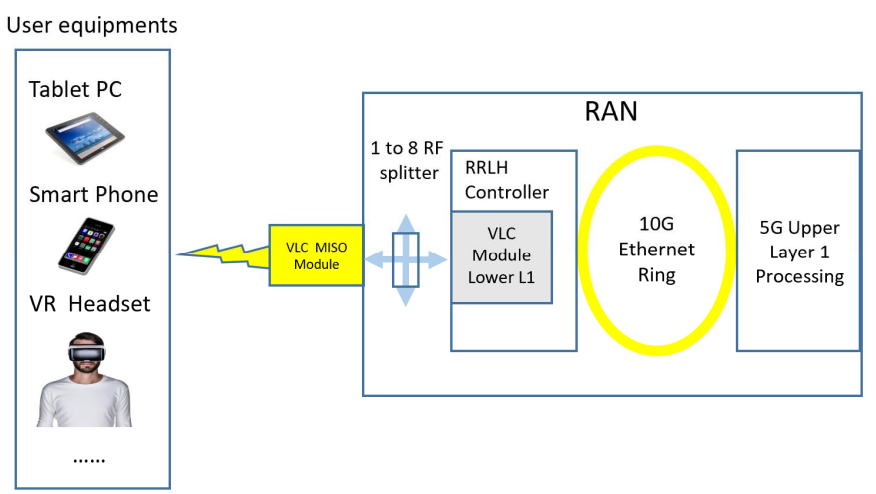

Fig. 1. Downlink scheme of 5G NR integration with VLC diagram

There are two issues integrating 5G NR with VLC that can be summarized as follows. First, VLC as an intensitymodulated/direct-detection (IM/DD) optical wireless system only accepts real-valued signals. However, the $5 \mathrm{G}$ NR baseband signal is complex-valued, therefore, a complex to real conversion is necessary.

Second, the modulation bandwidth of the VLC system by using commercial LEDs is limited to a few megahertz [10], therefore, the output 5G NR signal has to be adjusted for respecting this limitation. In order to address these two issues, we made the following adjustments: A) $5 \mathrm{G}$ NR modulation adaptation for VLC; B) $5 \mathrm{G}$ NR frame structure adaptation for VLC.

\section{A. 5G NR modulation adaptation for VLC}

This adaptation is aimed at generating real-valued $5 \mathrm{G}$ NR baseband signals. QPSK OFDM modulation is used as an example of this experiment.

In frequency domain only the positive frequency band carries the data symbols, which can be denoted by:

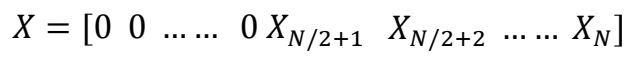

After N-point IFFT, we get the complex-valued 5G NR baseband signal $x(t)$ in time domain. Using the following relation, a real-valued signal $y(t)$ can be obtained.

$$
y(t)=x(t)+x^{*}(t)
$$

where $x^{*}(t)$ denotes the complex conjugate of $x(t)$.

Then a DC Bias-T is used to convert the bipolar real-valued signal $y(t)$ to strictly positive signal to meet IM/DD requirements [10]. The data symbols can be restored in the positive frequency band after a N-point FFT during the demodulation process.

Using the above adaptation only the Resource Blocks (RBs) on positive frequency can be scheduled for VLC signal transmission. Hence, the design of $5 \mathrm{G}$ NR Radio Resource Control (RRC) needs to be modified. However, compared with the conventional DC biased Optical OFDM (DCO-OFDM) and Asymmetrically Clipped Optical OFDM (ACO-OFDM) [11] transmission scheme, this modulation solution does not require large modifications to the physical layer component IFFT modulator. It only introduces one extra adder as described by equation (2)

\section{B. $5 G$ NR frame structure adaptation for VLC}

One 5G NR frame contains three main physical channels: Synchronization Signal/Physical Broadcast Channel (SS/PBCH), Physical Downlink Control Channel (PDCCH) and Physical Downlink Shared Channel (PDSCH) [12]. One 5G NR radio frame example is shown in Fig. 2.

The bandwidth of each channel has to be adjusted for adapting current VLC bandwidth of $10 \mathrm{MHz}$. Proposed modifications to each of the three channels are detailed below:

$\mathrm{SS} / \mathrm{PBCH}$ contains the basic synchronization signal and broadcasting information about essential VLC system configuration. According to TS 38.211 [12], one SS/PBCH block consists of $20 \mathrm{RBs}$ ( $1 \mathrm{RB}$ contains 12 contiguous subcarriers) in frequency domain and 4 contiguous OFDM symbols in time domain. In order to adapt to the VLC bandwidth, the subcarrier spacing is configured as $30 \mathrm{KHz}$. Thus, the SS/PBCH block occupies $20 \mathrm{RBs} * 12 * 30 \mathrm{KHz}=7.2$ $\mathrm{MHz}$ bandwidth for the proposed $5 \mathrm{G}$ NR frame structure.

PDCCH is to carry Downlink Control Information (DCI) that contains the demodulation configuration information such as number of RB, demodulation scheme and scheduling assignments. This information is essential for proper decoding of transmitted data. PDCCH could be configured with at least 1 Control Channel Element (CCE) and maximum 45 CCEs in frequency domain. In time domain, it occupies at most 3 consecutive OFDM symbols. For less than $10 \mathrm{MHz}$ bandwidth, 4 CCEs are allocated in PDCCH block.

$\mathrm{PDSCH}$ is allocated to the carrier payload of user service data. In PDSCH the resource allocation is flexibly scheduled by the base station with at least $1 \mathrm{RB}$ frequency resource and at least 1 OFDM symbol. Here, $25 \mathrm{RBs}$ for PDSCH are allocated, which occupy less than $10 \mathrm{MHz}$ bandwidth. 


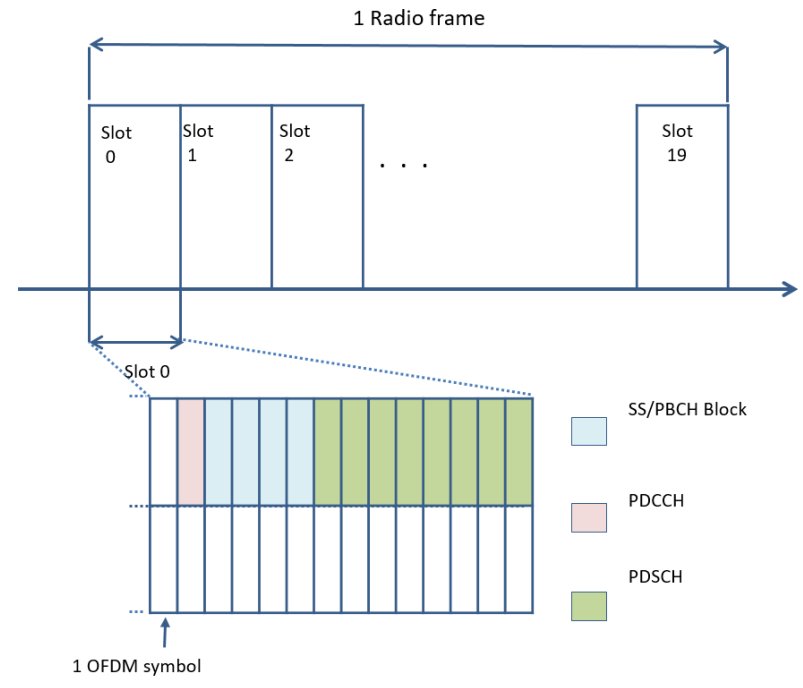

Fig. 2. 5G NR frame structure

As a result of the above facts and by adjusting the number of active RBs the $5 \mathrm{G}$ NR frame structure is suitable for VLC communication. In the next section we describe the implementation of the proposed downlink scheme on our $5 \mathrm{G}$ baseband testbed.

\section{EXPERIMENTAL TESTBED AND IMPLEMENTATION RESULTS}

\section{A. Experimental testbed description}

The experimental testbed is shown in Fig. 3, which was built to demonstrate the operation and feasibility of the proposed architecture. A personal computer (PC) and USRP act as local server and RRLH controller, respectively. A TP-Link Gigabit Ethernet Media Converter connects PC and USRP 2950R [13]. The USRP 2950R assembly with low frequency daughter boards (LFTX/LFRX) [14] is used to interface with the VLC front-end.

In the VLC front-end, the Bias-T inserts a $12 \mathrm{~V}$ DC voltage into the $5 \mathrm{G}$ NR signal to bias the LEDs in the linear range. At the receiver we used a Si avalanche photodetector (Hamamatsu APD C5331-11) to convert the received light intensity into an electrical signal. More details on VLC font-end parameters are seen in Table I.

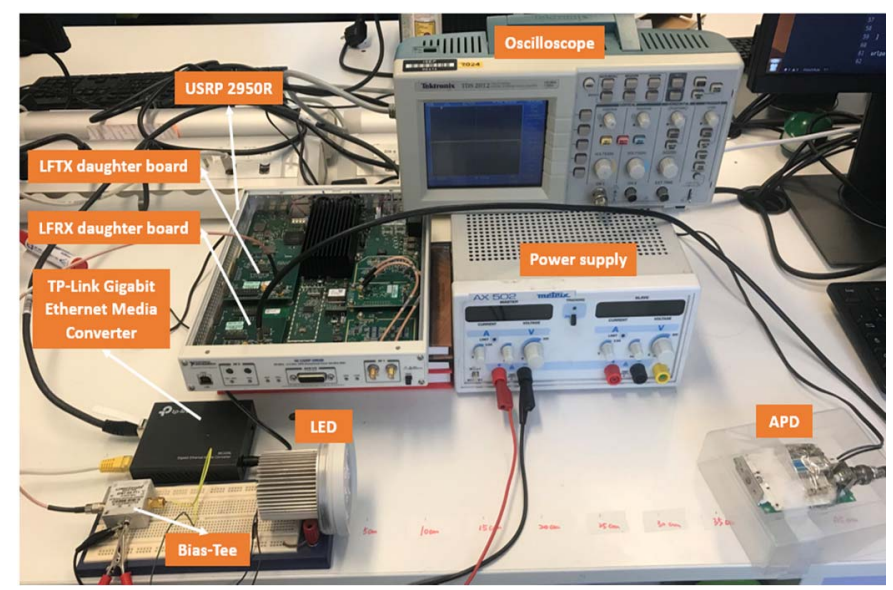

Fig. 3. Experimental testbed based on USRP 2950R
TABLE I. THE KEY PARAMETERS IN IMPLEMENTATION

\begin{tabular}{|l|l|}
\hline \multicolumn{1}{|c|}{ Parameter } & \multicolumn{1}{c|}{ Value } \\
\hline Input voltage & $12 \mathrm{~V}$ \\
\hline Operating current & $0.3 \mathrm{~A}$ \\
\hline Half-intensity beam angle & $30^{\circ}$ \\
\hline Photosensitivity & $0.42 \mathrm{~A} / \mathrm{W}$ \\
\hline
\end{tabular}

The signal flow graph in the testbed is illustrated in Fig. 4. The PC handles the signal processing for both transmitter and receiver. 5G NR signal is encoded and modulated by PC. It is sent from a local server to USRP via high-speed Ethernet cable. Output signal from USRP is merged with a $12 \mathrm{~V}$ DC source before sending to LED. An APD sends the received light signal to USRP Rx-side. Then, the PC decodes the received digital signal from USRP after the ADC conversion.

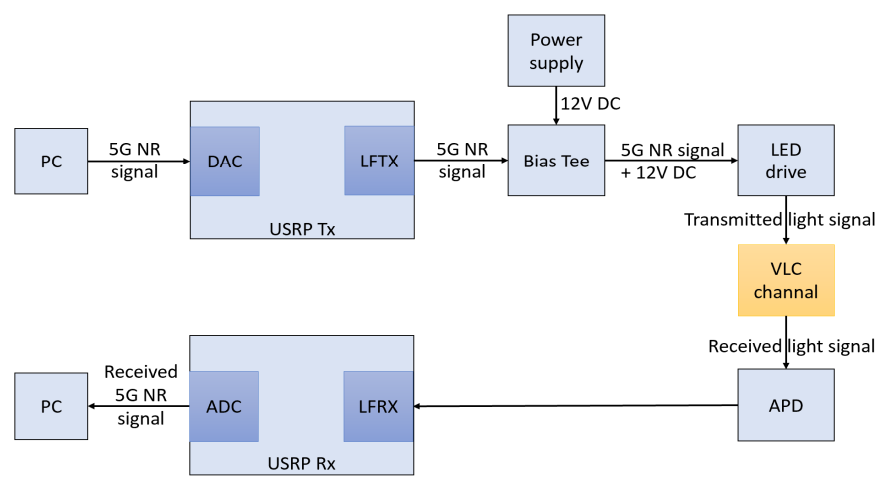

Fig. 4. 5G NR signal flow graph

\section{B. Implementation results}

In this paper, the communication performance is evaluated by measuring the EVM at different visible light transmission distance (from $5 \mathrm{~cm}$ to $55 \mathrm{~cm}$ ) as shown in Fig. 5. The proposed scheme with QPSK mapping offers a data rate of $14.4 \mathrm{M}$ bits/s and bandwidth of $7.2 \mathrm{MHz}$ with an EVM of less than $5 \%$ for a $55 \mathrm{~cm}$ free space transmission span.

The EVMs were measured by moving the APD away from the LED with $10 \mathrm{~cm}$ step distance. The values are $2.37 \%, 2.6$ $\%, 3.22 \%, 3.54 \%, 4.17 \%$, and $4.78 \%$ for distances of $5,15,25$, 35,45 and $55 \mathrm{~cm}$. At the same condition, in comparing with the LTE QPSK standard (limited at 17.5\%) in [15] and EVM of $15 \mathrm{~dB}(\sim 17 \%)$ in [16], our best EVM performance is $2.37 \%$.

From the constellation diagrams shown in Fig.6, we can see that higher constellation mapping might be accommodated. Our next step for the demonstration is to try higher-order constellation mapping to fully utilize the system's potential, such as using 64QAM and 128QAM, providing a higher bitrate of $43.2 \mathrm{M}$ bits/s and of $50.4 \mathrm{M}$ bits/s respectively. 


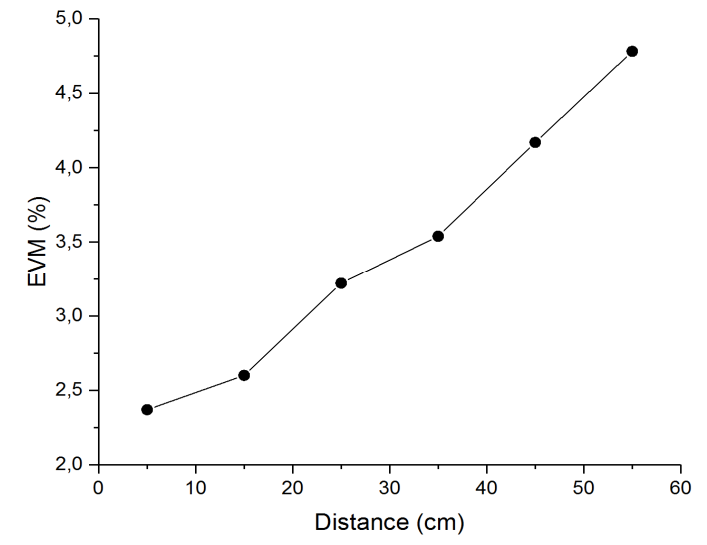

Fig. 5. EVM performance at different visible light transmission distance
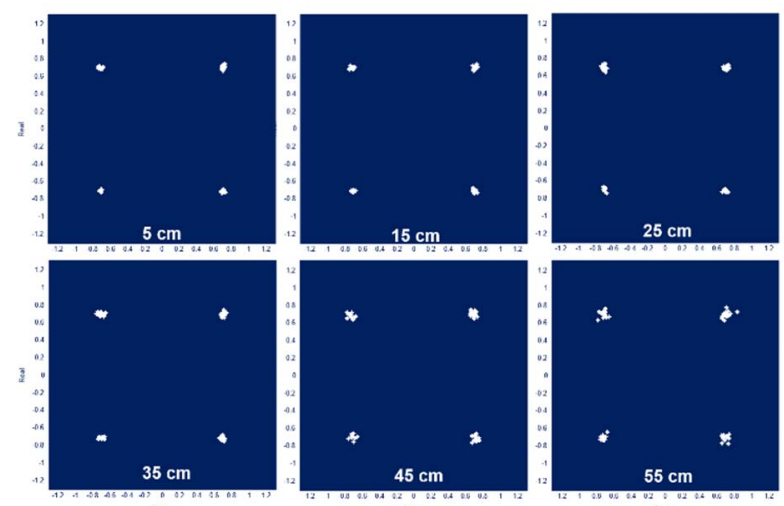

Fig. 6. The received constellation diagrams at different visible light transmission distance

\section{DISCUSSION AND FUTURE WORK}

In this paper we successfully performed the first test of $5 \mathrm{G}$ $\mathrm{NR}$ integration with VLC downlink. We focused on the study of the integration of 5G NR with VLC and feasibility of this scheme. The VLC performance improvement is not the priority of this paper. It is the first time a solution of integrating VLC with a $5 \mathrm{G}$ access network is presented, which is low cost, easy to install and compatible with the existing $5 \mathrm{G}$ standard.

The experimental results with a commercial LED lamp show the proposed scheme with QPSK mapping to offer a data rate of $14.4 \mathrm{M}$ bits/s and an EVM of less than $5 \%$ for a $55 \mathrm{~cm}$ free space transmission span. These results are implemented in the experimental environment and the feasibility is proven by measurements on the implemented testbed.

In the next step VLC performance improvement will be studied, which potentially could be optimized in two aspects: 1) optimizing the VLC front-end driver circuit. By using a postequalization front-end circuit and phosphorescent white VLC LEDs, a bandwidth of $151 \mathrm{MHz}$ and data transmission up to 340 $\mathrm{Mb} / \mathrm{s}$ have been achieved [10]; 2) addressing the baseband OFDM modulation problem. A high signal peak value for OFDM leads to a nonlinear distortion that causes serious degradation of system performance [17].
In the near future, the complete $5 \mathrm{G}-\mathrm{VLC}$ demonstration is to be built in the museum of carte à jouer in Paris with indoor high accurate location services [18].

\section{ACKNOWLEDGMENT}

The authors gratefully acknowledge the financial support of the EU Horizon 2020 program towards the Internet of RadioLight project H2020-ICT 761992.

\section{REFERENCES}

[1] Zvanovec, S., Chvojka, P., Haigh, P. A., \& Ghassemlooy, Z. (2015). Visible light communications towards 5G. Radioengineering, 24(1), 1-9.

[2] Feng, L., Hu, R. Q., Wang, J., Xu, P., \& Qian, Y. (2016). Applying VLC in $5 \mathrm{G}$ networks: Architectures and key technologies. IEEE Network, 30(6), 77-83.

[3] Bangerter, B., Talwar, S., Arefi, R., \& Stewart, K. (2014). Networks and devices for the $5 \mathrm{G}$ era. IEEE Communications Magazine, 52(2), 90-96.

[4] Wang, C. X., Haider, F., Gao, X., You, X. H., Yang, Y., Yuan, D., \& Hepsaydir, E. (2014). Cellular architecture and key technologies for 5G wireless communication networks. IEEE Communications Magazine, 52(2), 122-130.

[5] W. Li, Y. Zhang, L. Huang, C. Maple and J. Cosmas, "Implementation and co-simulation of hybrid pilot-aided channel estimation with decision feedback equalizer for OFDM Systems", IEEE Transactions on Broadcasting, vol. 58, no. 4, pp. 590-602, Dec 2012

[6] W. Ding, et al., A hybrid power line and visible light communication system for indoor hospital applications, Comput. Industry (2015), http://dx.doi.org/10.1016/j.compind.2015.01.006

[7] L. SHI; X. ZHANG; A VLADIMIRESCU. OFDMA-TDM position scheme for VLC indoor positioning system, GDR SoC-SiP Paris France, 2018

[8] Loi n 2015-136 ("Loi Abeille"), JORF n0034 du 10 février 2015 page 2346, texte $\mathrm{n}^{\circ} 1$

[9] Cosmas, J., Zhang, Y., \& Zhang, X. (2017, May). Internet of Radio-Light: $5 \mathrm{G}$ Broadband in Buildings. In European Wireless 2017; 23th European Wireless Conference; Proceedings of (pp. 1-6). VDE.

[10] Li, H., Chen, X., Huang, B., Tang, D., \& Chen, H. (2014). High bandwidth visible light communications based on a post-equalization circuit. IEEE photonics technology letters, 26(2), 119-122.

[11] Aminikashani, Mohammadreza, Wenjun Gu, and Mohsen Kavehrad. "Indoor positioning with OFDM visible light communications." Consumer Communications \& Networking Conference (CCNC), 2016 13th IEEE Annual. IEEE, 2016.

[12] 3GPP TS 38.211 in line with R1-1801291 after RAN1-AH-1801 (201802), 3rd Generation Partnership Project; Technical Specification Group Radio Access Network; NR; Physical Channels and Modulation (Release 15).

[13] Ni.com. (2018). SPECIFICATIONS USRP-2950. [online] Available at: http://www.ni.com/pdf/manuals/374194d.pdf [Accessed 4 Jul. 2018].

[14] Kb.ettus.com. (2018). LFTX/LFRX - Ettus Knowledge Base. [online] Available at: https://kb.ettus.com/LFTX/LFRX [Accessed 4 Jul. 2018].

[15] 3GPP TS 36.101 version 14.3.0 Release 14, Table 6.5.2.1.1-1

[16] LIN, Bangjiang, TANG, Xuan, GHASSEMLOOY, Zabih, et al.Experimental demonstration of an indoor VLC positioning system based on OFDMA. IEEE Photonics Journal, 2017, vol. 9, no 2, p. 1-9.

[17] ZHANG, Hua, YUAN, Ying, et XU, Wei. PAPR reduction for DCOOFDM visible light communications via semidefinite relaxation. IEEE Photon. Technol. Lett, 2014, vol. 26, no 17, p. 1718-1721.

[18] COSMAS, John, MEUNIER, Ben, ALI, Kareem, et al. 5G Internet of radio light services for Musée de la Carte à Jouer. In : LIFI Congress (GLC), 2018 Global. IEEE, 2018. p. 1-6. 